\title{
NOTAS SOBRE EL DESARROLLO DEL CONCEPTO DE CONFLICTO AMBIENTAL ONTOLÓGICO
}

\author{
JeRÔNIMO BAsILIO SÃo MATEUS ${ }^{1}$ \\ Investigador predoctoral en formación \\ Centre d'Estudis de Dret Ambiental de Tarragona (CEDAT) \\ Universitat Rovira i Virgili \\ Jeronimo.basilio@urv.cat
}

RESUMEN: En el artículo analizo el desarrollo de la idea de conflicto ambiental ontológico con el objetivo de demostrar su autonomía conceptual y la necesidad de tratarla como una categoría propia en filosofía política y en derecho. Sitúo la formación del referido concepto dentro de los postulados teóricos de lo que se denomina giro ontológico (ontological turn) en antropología y filosofía política. Argumento que esta línea de la antropología contemporánea posee herramientas metodológicas y conceptuales que pueden ser muy útiles en el ámbito jurídico para tratar de conflictos relacionados con lo que se denomina diferencias inconmensurables (Povinelli). Sostengo que, en materia ambiental, muchos de los conflictos de intereses ocurren en un nivel profundo, sobre la realidad misma de la cosa objeto del conflicto, y que, por lo tanto, deben ser tratados como conflictos ontológicos (Blaser). Al final, procuro establecer criterios hermenéuticos aplicables a la gestión de esta clase de conflicto ambiental.

RESUM: A aquest article s'analitza el desenvolupament de la idea de conflicte ambiental ontològic amb l'objectiu de demostrar la seva autonomia conceptual i la conveniència de tractar-la com una categoria pròpia a la filosofia política i al dret. Situo la formació d'aquest concepte dins dels postulats teòrics del que

\footnotetext{
${ }^{1}$ Becario del Ministerio de Economía y Competitividad - España. Ayudas para Contratos Predoctorales, 2014. Investigación conducida en el Marco del Proyecto Constitución Climática Global: Gobernanza y Derecho en un Contexto Complejo (DER2016-80011-P. Cofinanciado por MINECO/FEDER, UE).
} 
s'anomena gir ontològic (ontological turn) a antropologia i filosofia política. Argumento que aquesta línia de l'antropologia contemporània ofereix eines metodològiques i conceptuals que poden ser molt útils a l'àmbit jurídic per tractar conflictes relacionats amb això que s'anomena diferències incommesurables (Povinelli). Sostinc que, en matèria ambiental, molts dels conflictes d'interessos ocorren a un nivell profund, sobre la realitat mateix de la cosa objecte del conflicte, i que, per tant, han de ser tractats com conflictes ontològics (Blaser). Al final, procuro establir criteris hermenèutics aplicables a la gestió d'aquesta classe de conflicte ambiental.

ABSTRACT: In this article I analyze the development of the concept of ontological environmental conflict with the aim of demonstrating its conceptual autonomy and the need to deal with it as category of its own within political philosophy and law. I place the evolving definition of the term within what in anthropology and political philosophy is known as the ontological turn theory. I argue that this field of contemporary anthropology has its own methodological and conceptual tools, which can be useful within the legal sphere to deal with conflicts regarding incommensurable differences (Povinelli). I also claim that conflicts of interest related to the environment usually occur at a deep level, over the reality of the object underlying the conflict; therefore, they should be addressed as ontological conflicts (Blaser). Finally, I try to establish hermeneutic criterion for managing this kind of environmental conflicts.

PALABRAS CLAVE: Giro ontológico - Conflictos ambientales Inconmensurabilidad - Latour - Antropología simétrica.

PARAULES CLAU: Gir ontològic - Conflictes ambientals Incommensurabilitat - Latour - Antropologia simètrica

KEY WORDS: Ontological Turn - Environmental conflicts - incommensurability

- Latour - Symmetrical anthropology

SUMARIO: I. Introducción. II. Ejemplos de conflictos ambientales ontológicos. III. La crítica de la división naturaleza-cultura y el giro ontológico en la antropología contemporánea. 1. 
Crítica a la división naturaleza-cultura. 2. Giro ontológico en la antropología contemporánea. IV. Ontología política, ecología política y cosmopolítica. V. Autonomía conceptual y principios orientativos para el tratamiento jurídico de los conflictos ambientales ontológicos. 1. Autonomía conceptual de los CAO. 2. Criterios de identificación y principios orientativos. VI. Conclusiones. VII. Bibliografía

\section{INTRODUCCIÓN}

El objetivo del artículo es tratar de una clase particular de conflictos ambientales en los cuales no hay acuerdo sobre qué es la $\operatorname{cosa}^{2}$ objeto del conflicto porque una de las partes percibe o enuncia seres o relaciones que están ocultas para la otra parte. Hay dos modelos básicos: a) cuando una de las partes atribuye predicados no usuales a algún elemento natural ${ }^{3}$, por ejemplo, atribuirle agencia o intencionalidad a un río, una montaña o un animal; b) cuando una de las partes explica la controversia a través de elementos no directamente observables, como espíritus, dioses, etc.

De los dos modelos presentados se puede inferir que este tipo de conflicto suele ocurrir cuando cada parte posee ontologías -es decir, una distribución de los elementos y de las relaciones que identifica en el mundo- muy diversas. Por ejemplo, un conflicto entre el naturalismo, propio de la modernidad occidental, y algún tipo de animismo o perspectivismo, propio de los pueblos originarios americanos. En estos casos, si no hay acuerdo ni sobre qué es la cosa misma objeto de la controversia, ¿cómo llegar a una solución que contemple los intereses de las dos partes, por lo general el Estado/iniciativa privada, por una parte, y las comunidades indígenas o religiosas, por otra? ¿O, para poner un ejemplo concreto, cómo debe el Estado analizar el carácter sagrado de un determinado "recurso natural" en conflicto?

\footnotetext{
${ }^{2}$ La palabra cosa, en el artículo, es utilizada en el sentido que Latour le atribuye a partir del sentido etimológico de la palabra inglesa thing, o de su origen latino res, como algo que "conlleva siempre una discusión en el seno de una asamblea" (Latour, 2012b: 396). Se emplea en sustitución de la palabra objeto porque este lenguaje epistemológico conlleva determinados contenidos que se quiere evitar antes del debate público (por ejemplo, inercia, objetividad, pasividad). Afirmar que algo es un "objeto" ya es definirlo, atribuirle determinadas propiedades y negarle otras.

${ }^{3}$ Como se verá a lo largo del texto, utilizar la palabra natural para referirse a los elementos del mundo no creados por el hombre es problemático en el contexto de las discusiones sobre giro ontológico; sin embargo, en algunos casos la utilizo en beneficio de la claridad del texto. En el lenguaje del libro de Latour An Inquiry into the modes of Existence (AIME), el término apropiado sería líneas de fuerza o linajes, seres del modo reproducción [REP] (Latour, 2013: 91).
} 
La tesis defendida en el artículo es que esta clase de conflictos ambientales calificados de ontológicos $(\mathrm{CAO})^{4}$ porque se basan en una divergencia sobre las cosas que existen en el mundo - tienen autonomía conceptual, fundada en unas características propias distintas de las de los conflictos ecológicos de carácter distributivo (Martínez Alier, 2011: 88) que deben ser consideradas por la filosofía política, y en su regulación jurídica. Argumento que las soluciones basadas en el reconocimiento de "derechos culturales" a colectivos extramodernos ${ }^{5}$ son insuficientes para tratar de problemas ontológicos. Se argumenta, en este sentido, la necesidad de desarrollar una metodología de identificación de esta clase de conflictos, así como una serie de principios jurídicos aplicables a la solución de los casos llevados a juicio.

La estructura básica del argumento desarrollado en el artículo es la siguiente:

(a) La ontología occidental moderna, que divide el mundo en dos polos estancos y opuestos - por una parte, la naturaleza y, por otra, las culturas, considerando el primero el dominio de la certeza, de la inercia, del conocimiento objetivo y estable, y el otro el dominio de la subjetividad, la controversia, la agencia y la intencionalidad-, es incapaz tomar en consideración modelos ontológicos distintos, como pueden ser el animismo, el totemismo o el analogismo (Descola, 2012), porque ella misma es apenas uno de los modelos ontológicos posibles (naturalismo), una forma de ordenación pública de lo que es la realidad observada, y no compartimentos autónomos objetivos (Latour, 2012b: 97).

(b) En antropología, la universalización del modelo ontológico occidental provoca la incapacidad de aprehensión efectiva del funcionamiento de

\footnotetext{
${ }^{4}$ Según me consta, la expresión conflicto ambiental ontológico fue probablemente empleada por primera vez por Mario Blaser (2013a).

${ }^{5}$ Utilizo aquí y en todo el trabajo la terminología de Latour y Viveiros de Castro en sustitución de términos como cultura o diferencias culturales por considerar que estas expresiones no reflejan el grado de diferencia que existe entre los diversos colectivos. Esta última palabra, propuesta por el primero de los antropólogos citados (Latour, 2012b: 389), permite reconocer que las llamadas diferencias culturales son en verdad diferencias de articulación de todos los elementos del mundo, y no solamente de los llamados elementos sociales o culturales. Un colectivo es, por lo tanto, un conjunto articulado de elementos humanos y no humanos que no necesariamente está dividido entre las dos categorías del naturalismo moderno: naturalezacultura. El prefijo extra es propuesto por el segundo antropólogo en sustitución del no de "no modernos" para denotar una exterioridad a la modernidad, alejando posibles connotaciones evolucionistas en la otra expresión (Viveiros de Castro, 2016: 3).
} 
otros colectivos. En política, la misma universalización da lugar a modelos reduccionistas incapaces de dar cuenta de esta clase de diferencia radical (Povinelli, 2001). En derecho, una mala antropología puede generar injusticia.

(c) El multiculturalismo y su contraparte necesaria, el mononaturalismo, (Viveiros de Castro, 2014: 347), como productos del modelo ontológico occidental moderno, son incapaces, por lo tanto, de presentar buenas soluciones para la gestión de los CAO porque las diferencias ontológicas no pueden ser reducidas a meras diferencias entre culturas.

(d) Como alternativa, en primer lugar es necesaria una antropología que explique las diferencias radicales a partir de lo que en verdad son: diferencias sobre lo que existe en el mundo y la forma en que se interrelaciona, y no de carácter meramente epistemológico, diferentes percepciones sobre una misma realidad.

(e) En segundo lugar, es necesaria una política que sea capaz de gestionar problemas ontológicos, por lo que trato de algunas de las propuestas de Latour, Stengers y Blaser.

(f) Por último, el derecho, por su capacidad de imponer reglas, debe ser capaz de crear mecanismos que posibiliten el reconocimiento de las diferencias radicales como precondición a la capacidad de gestionar conflictos de esta naturaleza.

Esbozado el argumento en el párrafo anterior, el artículo se divide en cuatro partes: la primera está dedicada a la exposición de algunos casos concretos de CAO con la finalidad de delimitar el problema de forma precisa; en la segunda analizo la crítica a la división naturaleza-cultura en la antropología contemporánea introduciendo el concepto de giro ontológico; en la tercera trato de los reflejos para la filosofía política, del giro ontológico en antropología, a partir de los conceptos de ontología política (Mario Blaser), ecología política (Bruno Latour) y cosmopolítica (Isabelle Stengers); y en la última busco extraer criterios de identificación de los $\mathrm{CAO}$, así como enumerar los principios hermenéuticos que deberían informar la actuación del Estado en estos casos, tanto en la vía administrativa como en la contenciosa. 


\section{EJEMPLOS DE CONFLICTOS AMBIENTALES ONTOLÓGICOS}

Antes de iniciar el análisis, presento tres casos concretos de CAO con la finalidad de delimitar el problema que se pretende tratar en el artículo. Los casos son: el del pueblo dongria kondh de Odisha, India; el de la comunidad Yshiro; y el de la comunidad indígena Yakye Axa, los dos últimos de Paraguay.

El primero de los conflictos surgió a partir de la propuesta de implantación de minas de bauxita en las colinas Niyamgiri, en el estado de Odisha, India, por parte de una empresa privada minera (Vedanta Resources), en consorcio con una empresa pública del mismo estado (Odisha Mining Corporation Limited), en el año $2004^{6}$. Las ramificaciones del conflicto son muchas, $y$, hasta la fecha, no ha sido solucionado definitivamente, a pesar de que la Corte Suprema de la India ya se pronunció en 2013. En el ámbito del artículo me interesa destacar el argumento de la comunidad en contra de la implantación de la minería en la zona. De acuerdo con la cosmología propia de este pueblo, las colinas de Niyamgiri son la morada de un dios y ancestro (Niyamraja), lo que imposibilitaría la implantación de minas en la zona. La confrontación entre descripciones del mismo espacio físico es evidente: por una parte, para la empresa es una colina inanimada, mayormente desocupada, con una reserva de bauxita estimada en mil millones de dólares; por otra, para la comunidad es un bosque poblado de seres espirituales y habitado por un dios ${ }^{7}$.

Se trata de un conflicto ontológico porque no hay acuerdo sobre qué es la cosa misma objeto del conflicto, puesto que la comunidad local atribuye al lugar propiedades no reconocidas por la otra parte. En este caso, surge la siguiente cuestión: ¿existe algún elemento exterior, reconocido y aceptado por las dos partes capaz de poner fin a la controversia? ¿Hay algún elemento común, aceptado por ambas partes, capaz de mostrar de forma absoluta cuáles son todas las propiedades de la cosa objeto del conflicto? ¿Es la ciencia capaz de servir como este tercer intermediario?

El segundo ejemplo versa sobre un programa de caza sostenible en una región habitada por la comunidad Yshiro, en el noreste del país, junto al río Paraguay.

\footnotetext{
${ }^{6}$ Un resumen del caso puede encontrarse en el informe realizado en el ámbito del proyecto EJOLT (Cirone, 2015).

7 Para un análisis antropológico del conflicto se puede consultar, entre otros, el trabajo de Hazarika (2016).
} 
El conflicto en cuestión tiene su origen en las restricciones a la caza de tres especies de animales por parte del Gobierno paraguayo: carpincho (Hydrochoerus hydrochaeris), yacaré (Caiman sp.) y anaconda (Eunectes notaeus) (Blaser, 2009a: 81). Para atender las demandas de la comunidad para la caza de los referidos animales, el Gobierno del país implanta un programa de "caza sostenible" por el que concede permisos de caza comercial con la condición de que el número de animales se mantenga estable, de forma que se garantice la "sostenibilidad" de la actividad. El conflicto surge porque los Yshiro tienen un sistema diferente de mantener el equilibrio de su relación con los animales. Mientras que el Estado tomaba como indicadores de la sostenibilidad de la práctica exclusivamente la cantidad de animales cazados, los Yshiro consideraban que el número de animales no era el único factor relevante, porque el equilibrio con los animales tiene que establecerse a través de relaciones de reciprocidad que son interpersonales, incorporando, por ejemplo, elementos como la distribución de los beneficios de la caza entre la comunidad. Por esta razón, la federación que representaba al pueblo pedía exclusividad de la intermediación entre cazadores y comerciantes de las pieles. Su petición se fundaba en el conocimiento de los konsaho (chamanes), que son los intermediarios en la relación entre especies.

Como observa Blaser (2009a: 89), en este caso el conflicto es de naturaleza ontológica, puesto que el "medio ambiente" de las iniciativas gubernamentales no es lo mismo que el yrmo de los Yshiro. En el primer caso se trata de un espacio libre de agencia y de intencionalidad con el cual la única relación posible es de tipo sujeto-objeto; en cambio, para los Yshiro, constituye un mundo poblado de seres animados que genera relaciones sujeto-sujeto.

Mientras que las "creencias" del pueblo no afectaron a los estándares de conservación oficial, el Gobierno aceptó su idea de conservación. Sin embargo, cuando el número de animales empezó a disminuir, el Gobierno pasó a ignorar la gestión realizada por el pueblo e hizo uso de la fuerza policial para controlar la caza, aunque las autoridades Yshiro mantenían su posición en relación con el equilibrio de sus prácticas (Blaser, 2009a: 97). El discurso oficial entonces cambió y se comenzó a acusar a la federación de utilizar las cuestiones culturales para beneficiarse. 
El último de los ejemplos es un caso bastante conocido llevado a la Corte Interamericana de Derechos Humanos en 2003 —cuya sentencia fue dictada el 17 de junio de 2005- en el que las partes fueron la comunidad indígena Yakye Axa y el Estado de Paraguay. La cuestión de fondo era la delimitación de las tierras ancestrales de la comunidad. Esta última alegó que el Estado de Paraguay había vulnerado el derecho a la propiedad ancestral del pueblo al no delimitar sus tierras. La naturaleza ontológica del conflicto en cuestión se basa en la "relación especial" del pueblo con la tierra. Como quedó claro en la sentencia a partir de los peritajes antropológicos realizados, la relación de la comunidad con el territorio incluye aspectos inmateriales y sagrados (Corte IDH, 2005: 10), y no era solamente una relación de subsistencia material. La Corte aplica el artículo 13 del Convenio 169 de la OIT como criterio interpretativo del derecho a la propiedad (párrafos 135 y 136) ${ }^{8}$, incorporando los elementos "culturales" de la relación con la tierra a la hora de analizar la extensión de los daños sufridos por la comunidad.

En los tres casos no hay un acuerdo sobre qué es la cosa misma objeto del conflicto, puesto que estaban en contraposición diferentes ontologías. Por una parte, el Estado, con su naturalismo moderno, que divide el mundo entre varias culturas y una única naturaleza, despersonalizada, inerte, incapaz de actuar o sin intencionalidad; por otra, la perspectiva animista de las tres comunidades indígenas, para las cuales la relación con los elementos "naturales" es de orden intersubjetivo.

El problema central del artículo reside en cómo resolver esta clase de conflictos teniendo en cuenta los contenidos descriptivos de los enunciados de ambas partes. En seguida veremos por qué la solución que reduce todas las diferencias a diferencias culturales —claramente la visión expresada en la

\footnotetext{
8 "135. La cultura de los miembros de las comunidades indígenas corresponde a una forma de vida particular de ser, ver y actuar en el mundo, constituido a partir de su estrecha relación con sus territorios tradicionales y los recursos que allí se encuentran, no sólo por ser estos su principal medio de subsistencia, sino además porque constituyen un elemento integrante de su cosmovisión, religiosidad y, por ende, de su identidad cultural. 136. Lo anterior guarda relación con lo expresado en el artículo 13 del Convenio No. 169 de la OIT, en el sentido de que los Estados deberán respetar 'la importancia especial que para las culturas y valores espirituales de los pueblos interesados reviste su relación con las tierras o territorios, o con ambos, según los casos, que ocupan o utilizan de alguna otra manera, y en particular los aspectos colectivos de esa relación"”.
} 
sentencia del último caso- es insuficiente para comprenderlos y tratarlos de forma plena.

\section{LA CRÍTICA DE LA DIVISIÓN NATURALEZA-CULTURA Y EL GIRO ONTOLÓGICO EN LA ANTROPOLOGÍA CONTEMPORÁNEA}

El objetivo de este apartado es contextualizar lo que, en la antropología contemporánea, se denomina giro ontológico, presentando sus principales presupuestos teóricos, así como las consecuencias metodológicas para la investigación etnográfica. Este apartado servirá de introducción para el siguiente, en el que veremos algunas de las consecuencias políticas de esta forma de pensar y hacer antropología. La exposición se centrará en dos temas diferentes: 1) en primer lugar, presento la crítica de algunos antropólogos contemporáneos, principalmente Bruno Latour, a lo que dicho autor denomina "constitución moderna" (Latour, 2012a: 33), es decir, la división del mundo entre naturaleza y cultura; 2) en segundo lugar, comento los efectos metodológicos del giro ontológico en la antropología contemporánea.

\section{Crítica a la división naturaleza-cultura}

Cuando el antropólogo trata los modos de ordenar el mundo muy diferentes, de lo que Latour denomina la constitución moderna ${ }^{9}$, es decir, la división de todo lo observable en dos categorías con unas propiedades muy específicas no comunicables, tiene que adoptar un método para acercarse y entender la experiencia objeto de investigación. Aquí hay dos opciones fundamentales, que después se despliegan en otras tantas perspectivas metodológicas: explicar el dato etnográfico desde su ontología propia (del antropólogo, suponiendo que es una persona ajena a la cultura investigada) utilizando categorías básicas como social, cultural, natural, relaciones de poder, parentesco, propiedad y, la más amplia de todas, simbólico; o llevar a cabo lo que Latour denomina

\footnotetext{
${ }^{9}$ Latour adopta dicha expresión para designar la forma moderna de ordenar todos los elementos del mundo en dos polos: la sociedad, y la naturaleza. Utiliza de manera intencionada la palabra constitución, retirada del ámbito jurídico, para denotar el carácter construido y normativo de la referida separación, en contraste con los intentos de naturalizar algo que es exclusivamente epistemológico (Latour, 2012a: 34).
} 
irreduccionismo ${ }^{10}$, es decir, seguir de manera experimental la red de relaciones que produce y sostiene cualquier elemento de un colectivo dado. En otras palabras, practicar un empirismo radical: nada más allá de la experiencia, pero tampoco nada menos que la experiencia (Latour, 2013: XXV).

Si la última opción es la elegida, el antropólogo deberá estar abierto a entrar en el mobiliario del mundo (Latour, 2012b) propio de su contexto etnográfico, abandonando algunas cosas, resignificando otras y, por último, teniendo que incorporar, quizás, otros entes al mundo que le es común. Sin embargo, con el movimiento, el antropólogo todavía no llevó a cabo necesariamente el giro ontológico en su práctica etnográfica porque es posible que todavía esté situando la diferencia que observa no en las cosas mismas, pero sí en el observador; puede ser que aún esté ante una forma diferente de ordenar el mismo mundo al cual tiene completo acceso. Según Wagner (1981: 142), el antropólogo todavía puede estar identificando "otras maneras de tratar nuestra propia realidad" porque todavía toma la división naturaleza-cultura como propiedades de la realidad y no como un proceso de ordenación propio de su cultura.

Aquí es donde Latour entra con una de sus principales contribuciones a la antropología y a la filosofía contemporáneas ${ }^{11}$. Toda la obra del filósofo puede explicarse a partir de una preocupación central, que él mismo reconoce (Latour, 2013: 18), que es la de discutir en la modernidad los métodos válidos de producción de conocimiento, de acceso a la realidad o de "veridicción". Su crítica se produce en diferentes niveles y desde diversas perspectivas, yendo desde la antropología de la producción de conocimiento científico hasta la filosofía de la modernidad e, incluso, en el último Latour, hasta la metafísica ${ }^{12}$.

\footnotetext{
${ }^{10}$ El principio de irreductibilidad (principle of irreducibility) es un postulado metafísico propuesto por el autor en la segunda parte de Pasteurization of France que puede ser entendido en diversos niveles. Para los efectos del artículo, es suficiente considerarlo como una metodología de las ciencias sociales según la cual la investigación de cualquier objeto debe ser realizada a partir de sus relaciones. Para Latour, siguiendo a Whitehead, la realidad está constituida por procesos continuos, y el proceso de individualización y aislamiento es un procedimiento cognitivo posterior que nunca capta la totalidad de lo que es la experiencia real (Latour, 1988: 153).

${ }^{11}$ Para una evaluación de la contribución de Latour a la filosofía contemporánea, se puede consultar principalmente el libro de Graham HARMAN (2009): Prince of Networks: Bruno Latour and Metaphysics.

${ }_{12}$ El texto principal en el que Latour desarrolla cuestiones metafísicas es An inquiry into the modes of existence (AIME) (Latour, 2013).
} 
La tesis central de Latour, en relación con el tema tratado, es que la división del mundo entre naturaleza y cultura, característica de la modernidad occidental, es fruto de un proceso de purificación de la experiencia concreta, la cual, incluso para los modernos, es eminentemente híbrida, constituida por relaciones entre humanos y no humanos. Para el autor, la modernidad se considera la única "cultura" capaz de decidir con propiedad qué es la naturaleza, objetiva, cuyo acceso es garantizado por el conocimiento científico, y qué es la cultura, la esfera de producción subjetiva. Por esa razón, la modernidad se considera la única sociedad capaz de trascender las representaciones de la realidad y de acceder a la realidad misma (Latour, 2012a: 145).

Como consecuencia, el moderno considera que todos los otros colectivos humanos que existen en el mundo, que todavía no se han modernizado, aún funcionan y se organizan sin diferenciar bien entre qué es la realidad objetiva y qué son las representaciones y los símbolos (construcciones mentales subjetivas) proyectados sobre la realidad. Latour critica esta división entre modernidad y "los otros" por considerar que también los modernos, en sus prácticas concretas de producción de conocimiento, no separan qué es naturaleza o qué es sociedad. Lo que se denomina sociedad en la modernidad es en verdad una red de prácticas que está constituida también por representaciones y asociaciones con no humanos, sin las cuales las principales instituciones de la modernidad no funcionarían. De ahí el título de su importante ensayo Nunca fuimos modernos (Latour, 2012a: 184).

Esta diferencia entre las prácticas concretas de producción de conocimiento y la descripción de tales prácticas dada por la modernidad es fruto, según el autor, de una falta de atención a los procesos de mediación y de traducción que están necesariamente presentes en cualquier forma de acceso al mundo, incluido el conocimiento científico. Para el autor, la modernidad priorizó lo que denomina "procesos de purificación", es decir, la actividad de separar, clasificar, constituir esencias y formular conceptos propia de la actividad científica, y, como consecuencia, se olvidó de los procesos de mediación y de la realidad híbrida de la experiencia misma (Latour, 2012a: 28). Según Latour, 
la atención a la práctica concreta de las ciencias ${ }^{13}$, en todas sus contingencias, aspectos materiales, procesos de mediación, dispositivos de traducción y cuestiones políticas e institucionales, permite desmitificar el valor absoluto que se le atribuye en la modernidad occidental como única forma de acceso legítimo a la realidad, lo que, como consecuencia, posibilita el reposicionamiento del valor epistémico de otras reivindicaciones de acceso a la realidad.

A través de una antropología del conocimiento científico en la que Latour aplica el método etnográfico para estudiar una institución clave de la modernidad como es el laboratorio, el filósofo logra demostrar que el resultado (el conocimiento científico) es inseparable de sus procesos de producción. La ciencia existe por sus prácticas. Los hechos son construidos en laboratorio a través de los instrumentos de mediación, y, por lo tanto, son productos y no cosas anteriores al mismo proceso de investigación, porque la experiencia real transciende de manera inmensurable los cortes y las reducciones realizados por cualquier proceso de traducción/producción de información. Obsérvese que la posición de Latour no es relativista o exclusivamente constructivista (Harman, 2009: 59). El autor no niega la realidad ni la verdad; su crítica está relacionada con la teoría de la verdad como correspondencia (Kuukkanen, 2011: 180) y con la alegación de un acceso a lo real sin mediación, lo que en el AIME Latour denomina straigh talk (Latour, 2013: 125). Para el autor, la objetividad es producida, y el conocimiento que resulta tiene valor no porque sea un reflejo de la realidad, sino porque la institución que lo produce a través de sus procedimientos controlados, que pueden ser seguidos y repetidos, es fiable. La ciencia es objetiva, pero es completamente dependiente de sus medios de producción. Los llamados hechos científicos son constructos humanos producidos en laboratorios mediante una serie de instrumentos tecnológicos sin los cuales sería imposible la práctica de la ciencia.

Esta antropología de los modernos permite al autor realizar algo notable en antropología: comparar modernos y extramodernos, destruir el abismo que

${ }^{13}$ Latour inicia su carrera académica como antropólogo que se dedica a investigar la producción de ciencia en el laboratorio. La crítica más filosófica de la modernidad surgirá más tarde e incorporará muchos de los resultados de su actividad como antropólogo que se ocupa de los estudios sobre la ciencia. 
separaba la modernidad, única poseedora de los medios de acceso a la realidad, de todos los otros que, según el discurso común, todavía se hallaban en la confusión, sin distinguir bien objetos naturales de realidades simbólicas, míticas o supernaturales (Wagner, 1981: 143). Se observa que los modernos, así como los extramodernos, también acceden al exterior a través de mediaciones diversas y que cada diferente instrumento de acceso transforma lo que es percibido y señala hacia un aspecto de la realidad; ambos viven en un mundo que está poblado de seres híbridos constituidos por relaciones que pueden ser seguidas.

Latour, entonces, a partir de su crítica a la modernidad, abre el espacio para la construcción de una metodología diferente en antropología. Una metodología que reconoce que determinados problemas etnográficos deben ser tratados como cuestiones ontológicas. En el siguiente apartado veremos en qué consiste esta "nueva" aproximación.

\section{Giro ontológico en la antropología contemporánea}

El giro ontológico, como movimiento de la filosofía contemporánea que tiene ramificaciones en diversas otras ciencias sociales - como la antropología, la política, la filosofía de la ciencia, etc.-, se basa en algunos presupuestos teóricos, explicados parcialmente desde la perspectiva de Latour en el apartado anterior, que pueden resumirse en los siguientes puntos: a) el conocimiento de la realidad se lleva a cabo mediante procesos de traducción en los que la información es constituida, o normativizada, a partir del mismo proceso y a través de los diversos intermediarios, que también son en cierto sentido regulatorias; b) como consecuencia de lo anterior, se reconoce que la ciencia moderna no es el único medio de acceder al mundo, porque la ciencia funciona a través de la creación de modelos abstractos para explicar fenómenos observados siempre a través de instrumentos y nunca directamente -estos modelos no son la realidad ni su reflejo, sino más bien modelos interpretativos que pueden ser muy complejos y precisos, pero nunca completos y absolutos, puesto que siempre dependen de su proceso de producción: la ciencia es todo el procedimiento y no solo el resultado, y en cierto sentido produce los hechos que explica-; c) esta forma de abordar la producción del conocimiento 
científico permite relativizar la amplitud de muchas de sus conclusiones, o, por lo menos, de los efectos indirectos para la filosofía de la ciencia y para la epistemología política (Latour, 2012b: 392), y, como consecuencia, es posible cuestionar uno de los postulados básicos del dualismo moderno: la reducción de la naturaleza a sus propiedades físicas observables; y d) si los modernos reconocen, a partir de la crítica a la mayor garantía que tenían de un acceso privilegiado al mundo real -la ciencia-, que también su acercamiento a la realidad es siempre mediado y que, por lo tanto, no hay una diferencia tan abismal entre sus prácticas de organización del colectivo y la de los extramodernos, se abre la posibilidad de realizar lo que Latour denomina antropología simétrica (Latour, 2012a: 155) a partir de un redireccionamiento de los problemas antropológicos a cuestiones ontológicas.

Como metodología de investigación en antropología, el giro ontológico significa una disposición del antropólogo a seguir la experiencia etnográfica a partir de lo que se le presenta, sin efectuar ninguna reducción o generalización a categorías externas al propio contexto (Holbraad y Pedersen, 2017). El antropólogo, ante una situación que desafía postulados básicos de su ontología, como pueden ser la linealidad del tiempo, la división entre personas y cosas, el principio de causalidad material o la distribución de las agencias, debe estar abierto a entrar en la ontología local sin la necesidad de usar teorías generales sobre la sociedad, sobre lo humano y sobre su forma de organización. El antropólogo realiza un procedimiento de relativización ontológica (Holbraad y Pedersen, 2017) de todo su pensamiento con la finalidad de seguir el rastro de la experiencia y el modo de ordenación del mundo que es propio del contexto etnográfico que estudia. De esta forma, toma en serio a sus informantes porque analiza su experiencia y su explicación de esta a partir de sus mismos términos (Hazarika, 2016: 1), sin llevar a cabo reducciones precipitadas. El antropólogo busca entonces romper con la tendencia de explicar datos etnográficos diferentes a través de categorías familiares.

Como los mismos autores observan, el recurso a la ontología en antropología tiene un carácter metodológico. El antropólogo no se preocupa por resolver cuestiones metafísicas, como la búsqueda de principios generales sobre la 
realidad, lo que hace es acceder al vocabulario propio de la metafísica como recurso epistemológico utilizado en la integración de los datos de la experiencia etnográfica (Holbraad y Pedersen, 2017: 4). El antropólogo reformula el problema epistemológico de cómo alguien percibe las cosas en un molde ontológico: ¿qué es lo que hay para ser visto, en primer lugar? (Holbraad y Pedersen, 2017: 5).

El giro ontológico, más que una ruptura con la tradición de pensamiento en antropología, es una radicalización de sus intuiciones iniciales sobre la necesidad de asumir la perspectiva del otro. La disposición del antropólogo para reconstituir todos los conceptos que utiliza, a partir de la experiencia etnográfica, permite crear unas herramientas analíticas bastante eficaces para describir y comprender los materiales que trata. Además, permite descolonizar la antropología y centrarla en cada realidad que investiga (Castro, 2010).

En este sentido, la utilización de este tipo de aproximación puede contribuir a la comprensión de los CAO porque permite crear un sistema de traducción de realidades muy diversas sin la necesidad de recurrir a los típicos artefactos del naturalismo moderno, como la reducción de toda articulación de experiencias del mundo ajenas al contexto occidental a formas simbólicas, míticas o representaciones.

En el siguiente capítulo veremos algunos de los efectos propiamente políticos de la adopción de dicha aproximación.

\section{ONTOLOGÍA POLÍTICA, ECOLOGÍA POLÍTICA Y COSMOPOLÍTICA}

En el ámbito político, el giro ontológico también posee un potencial importante en la creación de herramientas conceptuales más capacitadas para tratar de la clase de diferencias radicales que se analizan en el artículo. La dimensión política del giro ontológico puede resumirse en la expresión de Viveiros de Castro (2010) "autodeterminación ontológica", es decir, la capacidad de los colectivos de decir y de ver reconocido su derecho a habitar el mundo que realmente es el suyo, sin tener que reducir sus dimensiones de existencia, cuando haya necesidad de contactar con el Estado. En el presente apartado analizo los efectos del giro ontológico en filosofía política a partir del análisis de 
tres conceptos: ontología política (Mario Blaser), ecología política (Bruno Latour) y cosmopolítica (Isabelle Stengers).

De lo expuesto hasta ahora se puede afirmar que tratar toda la experiencia de los colectivos extramodernos en el mundo como "cultura", considerando todas sus afirmaciones sobre la realidad como simbólicas, es un modo inadecuado de conocer esta misma experiencia. Se les reconoce el derecho a la cultura, pero se les retira el derecho a la realidad, según la precisa expresión de Latour (2012b: 81). La palabra cultura, cuando se refiere a colectivos extramodernos, suele utilizarse para agrupar cosas tan distintas como hábitos alimentarios, técnicas de producción agrícola, festividades, relaciones familiares, formas de organización social..., comprendiendo incluso afirmaciones metafísicas, la ontología, la cosmología, su "(etno)"ciencia y religión propias... Cuando se utiliza para referirse a los modernos, sin embargo, la palabra no tiene la misma amplitud porque, dado que los modernos serían capaces de separar la realidad de sus representaciones, serían los únicos que tendrían una cultura y, asimismo, la capacidad de acceder a lo real a través de sus ciencias. Según esta visión, ningún colectivo extramoderno vive en verdad en la realidad porque ignora sus constituyentes básicos (leyes físicas, materia, los átomos, los genes), vive en representaciones o en proyecciones de sus aspectos interiores sobre el mundo físico (Descola, 2012: 135).

Las consecuencias políticas de la adopción de un modelo antropológico o de otro son obvias porque, si el Estado presupone que toda afirmación sobre la realidad que no tenga el aval de la ciencia moderna se aparta de lo que Blaser (2013) denomina "política racional", se la considerará, por lo tanto, de menor valor y, probablemente, no será tenida en cuenta cuando entre en conflicto con "problemas reales" (desarrollo, producción económica, alimentación, salud, etc.). Aquí se evidencia que la división entre el Occidente moderno, único colectivo con acceso a la realidad, y todos los demás pueblos, cuyo conocimiento, modos de relación y constitución del mundo son reducidos a "manifestaciones culturales", puede generar injusticias cuando el Estado tiene que gestionar un conflicto de intereses.

Tomando el ejemplo concreto de los dongria kondh, la versión considerada "real" es la de la empresa minera porque trata de "recursos minerales", 
"desarrollo económico"..., cosas tangibles. Esta descripción goza, por lo tanto, de un estatuto privilegiado, puesto que se refiere a la "naturaleza" real, mientras que los dongria kondh tendrían acceso solamente a representaciones de esta, en otras palabras, su descripción (ya no es propiamente una descripción, sino más bien una narrativa ficcional o mítica) se incluiría en el polo opuesto, la cultura (Hazarika, 2016: 14).

Como observa Blaser (2009b), el espacio de la política ordinaria está delimitado por lo que se considera racional, en un sentido bastante restrictivo del término, es decir, de lo que se considera que puede ser probado por la ciencia experimental. Todo lo demás, todo lo "irracional", solo tiene espacio en la esfera política dentro de la cultura. El valor que se atribuye a estos enunciados dentro del debate público es más bien cosmético o de una excentricidad tolerada mientras no comprometa cuestiones más importantes.

Como modelo alternativo para reflexionar sobre esa clase de problemas políticos, Blaser propone un modelo de filosofía política abierto al problema ontológico al que denomina ontología política y que se caracteriza por tres cambios: una sensibilidad y una problemática propias y una metodología de análisis crítico (Blaser, 2013a). La sensibilidad propia supone asumir un compromiso con el pluriverso, es decir, con el reconocimiento de que el acceso a la realidad es múltiple y que la realidad percibida depende de los medios utilizados. La consecuencia básica de esta sensibilización es que los modelos ontológicos distintos no pueden ser descartados de inmediato como irracionales, simbólicos o míticos, sino que deben ser integrados en el espacio público. Así pues, la problemática básica a la que se debe orientar el filósofo político es la forma mediante la cual son percibidas las diferentes realidades 0 , mejor aún, son constituidos los diferentes mundos, y de qué forma se relacionan y pueden convivir en el espacio público institucional. Como consecuencia, la metodología de análisis propuesta no se ocupa de buscar una definición última de la realidad a partir de la cual se definiría la esfera de lo público, sino que, por el contrario, amplía los problemas tratados en filosofía política, situando la cuestión de la naturaleza o del exterior como uno de los temas fundamentales a ser considerados y construidos. 
La ontología política, en este sentido, va más allá de los análisis de la economía ecológica o la ecología política porque, como observa Blaser (2009a), estas dos metodologías todavía trabajan dentro del binomio multiculturalismo-mononaturalismo. La filosofía política, resignificada a partir del giro ontológico, al romper con la unidad de la naturaleza, reposiciona las diferencias entre colectivos como diferencias entre naturalezas-culturas (Latour, 2012a).

En el último capítulo veremos cómo los referidos planteamientos influyen en el análisis político de los $\mathrm{CAO}$; veamos ahora las otras dos reformulaciones de la actividad política.

La propuesta de Latour para una nueva política surge de su crítica de la modernidad, como ya se ha visto en los apartados anteriores. Fundamentalmente, Latour (2012b: 79) retoma la crítica de Whitehead sobre la división del mundo en cualidades primarias (propiedades que están en las cosas, mensurables y objetivas, como la extensión o la textura) y cualidades secundarias (propiedades que solo son percibidas por la mente humana y, por lo tanto, subjetivas, como los colores, los olores, etc.) para explicar por qué las divisiones entre naturaleza-cultura, ciencia-política o sujeto-objeto son naturalizadas, consideradas simples reflejos de la realidad. Esto explica, según el autor, por qué la política normalmente transcurre al margen de cuestiones ontológicas. La constitución moderna, es decir, la forma de ordenación del espacio público en la modernidad, atribuye a la ciencia el papel exclusivo de definir qué es lo que existe; como resultado, a la política, como actividad subsecuente, le queda solamente la tarea de crear formas de gestionar el mundo previamente estabilizado y unificado por la ciencia. No hay, por ejemplo, dentro del debate público, espacio para ninguna controversia sobre qué es un río porque todas sus propiedades "reales" pueden ser establecidas de forma definitiva por las ciencias competentes (biología, geología, geografía) ${ }^{14}$. Todo lo demás son aspectos simbólicos, culturales, religiosos o míticos inapreciables como enunciados descriptivos. Contra esta posición, Latour argumenta que la actividad política es el espacio propio de construcción del mundo común, por lo

\footnotetext{
${ }^{14}$ Para un interesante análisis, basado en las teorías de Latour, de las posibles dimensiones de un río en concreto (el Ganges en la India), se puede consultar el trabajo de Elizabeth Ann McAnally (2007).
} 
que las cuestiones ontológicas constituyen una parte considerable de cualquier discusión pública, por lo menos cuando están en juego las referidas diferencias radicales.

Según esta visión, la política es reconfigurada como diplomacia (Latour, 2012b: 391), como un proceso de traducción de realidades inconmensurables cuya finalidad es posibilitar la convivencia, la cohabitación del mismo espacio físicoinstitucional. Su presupuesto fundamental es asegurar la posibilidad de enunciar plenamente la descripción del mundo en el que se vive, sin que haya previamente una reducción de todo enunciado con contenido descriptivo a enunciados míticos o simbólicos vaciados de realidad. La política como diplomacia no puede más presuponer una unificación previa de la naturaleza, universal dotada de las mismas propiedades, igualmente accesible; lo natural, lo social, la distribución de las agencias, las relaciones de sujeto-objeto o persona-cosa..., deben consensuarse en el proceso político y no tomarse como reflejos de una realidad objetiva anterior y previa, indiscutible y compulsoria.

La política, cuando reconoce como propia la necesidad de llegar a consensos incluso sobre cuestiones muy básicas, se reconfigura como cosmopolítica (Stengers, 2010), una política que reconoce que no trata específicamente de problemas humanos, sino de todo el cosmos - por lo menos, de esta parte del cosmos en que nos situamos, al que Stengers, Latour, Viveiros de Castro, tomando la idea original de James Lovelock, se refieren como Gaia-, y que, por otro lado, reconoce el carácter político, es decir, consensuado, colectivo y mediado, de cualquier acercamiento a la realidad exterior. O, para citar la explicación de Latour del concepto de Stengers: "The presence of cosmos in cosmopolitics resists the tendency of politics to mean the give-and-take in an exclusive human club. The presence of politics in cosmopolitics resists the tendency of cosmos to mean a finite list of entities that must be taken into account. Cosmos protects against the premature closure of politics, and politics against the premature closure of cosmos" (Latour, 2004).

La resignificación de la política como cosmopolítica permitiría, para utilizar la expresión de Latour, convocar nuevamente el colectivo para llegar a un consenso sobre el mundo común. Tanto Latour como Stengers desarrollan sus idearios políticos como formas de afrontar la crisis ambiental actual en el 
contexto del antropoceno (Stengers, 2015); sin embargo, el reposicionamiento de la relación modernidad-extramodernidad que los autores proponen seguramente también puede informar todos los conflictos relacionados con las referidas diferencias radicales.

\section{AUTONOMÍA CONCEPTUAL Y PRINCIPIOS ORIENTATIVOS PARA EL TRATAMIENTO JURÍDICO DE LOS CONFLICTOS AMBIENTALES ONTOLÓGICOS}

\section{Autonomía conceptual de los CAO}

Para finalizar el artículo, en este apartado trato de la autonomía conceptual y consecuente necesidad de tratamiento autónomo de los CAO en relación con otros tipos de conflictos ambientales. Además, presento algunos de los criterios que considero fundamentales para analizar dichos CAO.

Para verificar la autonomía conceptual de los CAO, es necesario en primer lugar saber en qué consisten los conflictos ecológicos o ambientales como categoría general. Una de las definiciones más simples, aunque completa, de los conflictos distributivos es la de Martínez Alier (2011: 108), para quien los conflictos ecológicos son aquellos que tratan sobre la distribución de los recursos o servicios ambientales o sobre la distribución de las cargas de producción de determinado bien o servicio ambiental. Son conflictos, por lo tanto, sobre los beneficios de la utilización de cualquier elemento de la naturaleza o sobre los residuos generados por su aprovechamiento económico.

Por la definición, es posible constatar que los CAO pueden considerarse un subtipo de conflicto ambiental, si bien no hay consenso sobre la extensión o las propiedades de la cosa objeto de conflicto. Por ejemplo, en el caso Masacres de Río Negro Vs. Guatemala, el hecho objetivo de no enterrar a sus muertos tiene para el pueblo maya efectos mucho más complejos que el mismo hecho para alguien ajeno a la comunidad. Además del sufrimiento emocional, algo más o menos común, no enterrar a los muertos conlleva problemas para toda la comunidad: tanto para los propios muertos, que no son enterrados en los lugares sagrados apropiados, como para los familiares, que no pueden cumplir con su obligación de alimentarlos ni con los rituales y las ofrendas comunes 
que se realizan después de la muerte. Por otro lado, la inundación provocada por la hidroeléctrica de Chixoy inundó los sitios sagrados de la comunidad en los que se realizaban las ceremonias fúnebres (párrafo 159 de la Sentencia de 4 de septiembre de 2012). Sin duda alguna, la inundación de tierras de cualquier persona o grupo de personas contra su voluntad provoca un daño evidente; sin embargo, este daño puede agravarse de forma importante cuando la relación que esta comunidad o persona establece con el espacio perdido es una relación que trasciende la mera explotación económica o disfrute estético o recreativo. La sacralidad del espacio físico cambia la naturaleza del conflicto ambiental porque una de las partes señala afectaciones que muy probablemente no puedan ser percibidas directamente por la otra. Una de las partes describe el conflicto en términos que son ajenos a la otra parte, como, por ejemplo, en el caso comentado, la pérdida del contacto con los guías espirituales del pueblo tras la inundación de los sitios sagrados (párrafo 87 de la Sentencia de 4 de septiembre de 2012).

Si se consideran los CAO como una clase de conflicto ambiental ordinario, sin tener en cuenta la naturaleza ontológica de la controversia de fondo, la solución probablemente será llevar a cabo un análisis de coste-beneficio de la obra que se pretende realizar en el que todos los enunciados considerados "irracionales" serán tomados como "aspectos culturales" cuya valoración deberá venir precedida de una traducción de estos valores a un lenguaje económico que pueda ser contabilizado, como, por ejemplo, a través de la idea de servicio ecosistémico.

Desde los estudios de economía ecológica y de ecología política ya se ha realizado una crítica de esta reducción de cualquier "valor" al lenguaje económico, como si este tipo de lenguaje fuera el único lenguaje imparcial u objetivo que pudiera servir para informar una decisión estatal. Como alternativa se propone la aceptación de un "pluralismo de valores inconmensurables" (Martínez Alier, 2011: 354) para contrastar el reduccionismo económico. Sin embargo, la solución propuesta desde la perspectiva de la ontología política es aún más radical porque cambia los términos en que sitúa la controversia. Considerar las diferencias entre los colectivos como una diferencia entre valores es mantenerse dentro de una perspectiva naturalista moderna según la 
cual lo que cambia es la valoración que se tiene del mundo y no el mundo objetivo. Este tipo de lenguaje, por lo tanto, todavía no es capaz de captar propiamente el modo de organización de estos otros colectivos porque, obviamente, para las comunidades indígenas de cualquiera de los casos vistos en el punto 2 no constituye una mera representación simbólica o un simple valor agregado al mundo natural, sino de una realidad tan tangible como cualquier elemento físico.

La autonomía metodológica y conceptual de los CAO surge, en este sentido, de la necesidad de crear mecanismos de traducción político-jurídicos de esta realidad inconmensurable.

\section{Criterios de identificación y principios orientativos}

De todo lo expuesto se deduce que los CAO deben gozar de una atención especial por parte del Estado. En este apartado sintetizaré algunos de los puntos clave a considerar a la hora de gestionar esta clase de conflictos, bien cuando el conflicto esté en fase no contenciosa, bien cuando ya haya derivado en una demanda judicial. Al final comentaré algunos dispositivos legales dirigidos al reconocimiento de la clase de diferencias radicales tratadas anteriormente.

En primer lugar, es necesario saber cómo identificar esta clase de conflictos ambientales. Blaser aporta algunas consideraciones importantes al respecto. Lo primero que señala el autor es que no se puede identificar un conflicto ontológico ambiental solamente mirando a las partes del conflicto. Es decir, no todo conflicto con presencia de pueblos indígenas es un conflicto ontológico. Lo importante, por lo tanto, no es la pertenencia a un grupo específico, sino sus prácticas concretas (Blaser, 2013a). El conflicto ontológico solo tiene lugar cuando una de las partes constituye su mundo de forma distinta, es decir, posee otra ontología y, como consecuencia, puede atribuir propiedades o establecer relaciones no usuales con un determinado elemento natural, o también convocar al conflicto o describirlo recurriendo a entes no observables científicamente. 
Identificada la existencia de un $\mathrm{CAO}$, ¿cuál deberá ser la actuación del Estado? No es posible realizar de forma abstracta un análisis concreto de la normativa aplicable, puesto que esta varía, evidentemente, en función de cada Estado, de los tratados internacionales que haya suscrito, de las fuerzas políticas involucradas en el conflicto, de los derechos constitucionales reconocidos, etc. El objetivo aquí, más que señalar normas concretas, es tratar de recopilar aquellos puntos generales sobre la naciente teoría de los conflictos ambientales ontológicos que deben ser considerados por los agentes públicos que se hallen ante un caso concreto. He seleccionado tres puntos importantes, que pueden considerarse criterios hermenéuticos generales sobre el tema:

(a) No vaciar de contenido los enunciados descriptivos de las partes. Esta es la premisa básica de cualquier política preocupada por cuestiones ontológicas. Si la experiencia del mundo de una de las partes es reducida a "aspectos culturales", ya se están valorando negativamente sus reivindicaciones.

(b) Garantizar que las partes puedan enunciar el conflicto del modo en el que lo experimentan. Es condición previa para discutir los términos de cualquier posible solución la posibilidad de expresar el conflicto en sus mismos términos y en todas sus dimensiones.

(c) El objetivo de la solución de un conflicto de esta naturaleza no debe ser llegar a un consenso sobre qué es la realidad misma, que entonces se impondría como solución obligatoria. Más bien, el objetivo de la ontología política es crear condiciones para que los diferentes mundos puedan mantenerse vigentes.

Todavía queda por realizar la traducción de estos objetivos políticos a un lenguaje jurídico. ¿Es el lenguaje de los derechos una herramienta eficaz en este sentido? La cuestión merece un análisis mucho más detallado del que es posible en este espacio; sin embargo, se pueden apuntar algunas breves cuestiones. Aunque la palabra derechos quizá no refleje del todo bien la relación que se establece entre determinado pueblo y su entorno natural cuando, por ejemplo, dicho pueblo mantiene relaciones de parentesco con determinados elementos naturales y sus sentimientos hacia el entorno son más bien de responsabilidad, el lenguaje de los derechos es una creación occidental 
que puede servir muy bien como garantía de esferas de autonomía personal y colectiva. Por lo tanto, puede ser interesante que el reconocimiento de las mencionadas diferencias radicales se lleve a cabo mediante la declaración de derechos. De cualquier forma, la mera declaración no es suficiente si no se tiene en cuenta una hermenéutica apropiada para abordar este tipo de conflictos. Es decir, la declaración de derechos no es suficiente si se considera que las reivindicaciones son absurdas, irracionales, irreales o dispensables, porque, en el conflicto de intereses y de derechos, el peso de la irrealidad siempre recaerá sobre la parte cuyo mundo no es accesible.

Para terminar, veremos algunos ejemplos de disposiciones o normas en las que el derecho intenta acercarse, de forma poco consciente, a esta clase de diferencias radicales desde diversas perspectivas. Comentaré tres dispositivos. El primer dispositivo importante es el artículo 13.1 del Convenio 169 de la OIT de 1989, cuyo texto es:

Artículo 13 1. Al aplicar las disposiciones de esta parte del Convenio, los gobiernos deberán respetar la importancia especial que para las culturas y valores espirituales de los pueblos interesados reviste su relación con las tierras o territorios, o con ambos, según los casos, que ocupan o utilizan de alguna otra manera, y en particular los aspectos colectivos de esa relación.

Dicho artículo es importante porque, por primera vez, la llamada "relación especial con las tierras o territorios" es reconocida en el derecho internacional en un documento con fuerza vinculante. De esta forma, el derecho internacional reconoce que la forma en que los pueblos indígenas se relacionan con la tierra puede ser muy distinta del patrón occidental. Este artículo es utilizado por la Corte Interamericana de Derechos Humanos como criterio de interpretación del derecho a la propiedad en algunos casos de conflictos indígenas, tal como ya se ha explicado en el punto 2.

La Declaración Americana sobre los Derechos de los Pueblos Indígenas de la Organización de los Estados Americanos (OEA) trata de manera separada los derechos culturales y a la integridad cultural (artículo XIII) y los derechos relacionados con la "espiritualidad indígena" (artículo XVI) en un importante paso, en el sentido de reconocer que lo que se entiende por espiritualidad de un pueblo trasciende el elemento cultural porque esta, denominada asimismo 
cosmovisión (expresión también utilizada de forma novedosa ${ }^{15}$ en la Declaración —artículos XIII.3, XV.5, XIX.1, XXIX.1-), es también una explicación de los elementos "naturales".

La última de las normas que citaremos es el artículo 3 de la orden interministerial de la República de Benín ${ }^{16}$. Lo interesante de esta regulación son las definiciones de "Forest of the ancestors", de "Forest of the gods or spirits" y de "Sacred Forest", respectivamente:

\section{CHAPTER II: Definitions}

Article 3: For the purpose of this decree, the following terms shall mean:

Forest of the ancestors: Any forest home to ancestors' spirits.

Forest of the gods or spirits: Any forest where one or several gods or spirits live.

Sacred Forest: Any forest home to several gods worshipped by the local population. It can be a hunting reserve, a forest of the ancestors, a burial forest, a forest of the gods or spirits, or a forest of secret societies [Republica de Benín, 2012)]. (La cursiva es nuestra).

Como se puede observar de las tres definiciones citadas, la orden interministerial entra realmente, gracias al vocabulario utilizado, en la ontología local porque no usa las habituales palabras condicionantes, que vacían los enunciados de su significación descriptiva. En ese sentido, las selvas de los ancestros son aquellas en las que los ancestros habitan, y no las que se consideran moradas de los ancestros. De la misma forma, los bosques de los dioses y espíritus son aquellos en los que estos seres viven (live), y no los que se consideran habitados, $\mathrm{y}$, por último, los bosques sagrados son los bosques habitados (home to) por dioses. Al no utilizar palabras que relativizan el contenido de las definiciones locales, el artículo comentado ${ }^{17}$ trasciende el $^{-1}$ lenguaje del relativismo cultural habitual del derecho y adopta un modelo

\footnotetext{
${ }^{15}$ En la Declaración de Naciones Unidas no se utiliza el término.

16 Concretamente, "INTERMINISTERIAL Ol ORDER $N^{\circ} 0121 / M E H U / M D G L A A T / D C / S G M / D G F R N / S A$ Setting the conditions for the sustainable management of sacred forests in the Republic of Benin".

${ }^{17}$ No sé si el redactado de la norma fue intencionado o bien fruto de la causalidad, puesto que en otros pasajes de la orden interministerial se utiliza el lenguaje del relativismo cultural, como, por ejemplo, en el artículo 2: "Subject to these rules are any spaces considered as habitats of several gods worshipped by the people".
} 
normativo que reconoce el pluriverso, en el sentido que he expuesto en el trabajo.

Para finalizar, cabe mencionar una tendencia mundial cuyos resultados concretos todavía están por evaluar, pero que sin duda constituye una innovación legislativa y jurisprudencial, al menos en el ámbito conceptual: el reconocimiento de personalidad jurídica a diversos ríos. Hasta el momento, hay por lo menos cuatro ríos reconocidos: el Te Awa Tupua en Nueva Zelanda; el Ganges y el Yamuna en la India; y el Atrato en Colombia. La primera norma reconoce que el Te Awa Tupua, también conocido como Whanganui, es una unidad viva e indivisible (subpart 2,12) que comprende en toda su extensión y los elementos "físicos y metafísicos". Se le reconoce personalidad jurídica plena (subpart 2, 14.1) (Nueva Zelanda, 2017). En el segundo caso, se trata de una decisión judicial de la Corte Suprema del estado de Uttarakhand, en el norte de la India, por la cual se declara que los dos ríos mencionados son personas jurídicas (legal person) con los mismos derechos que un ser humano (punto 5 de la parte dispositiva). En la motivación de la sentencia se observa que la Corte intenta, a través de la declaración, fomentar la conservación de ambos ríos. Mediante la misma decisión, la Corte nombra como representantes legales a una serie de entidades públicas y privadas (punto 3) (India, 2017). En el caso de Colombia, también se trata de una decisión judicial emitida por la Corte Suprema del país a través de la cual se considera el río como una "entidad sujeto de derechos" (República de Colombia, 2016). Dicha sentencia nombra diversos representantes legales entre los miembros del Gobierno y de las comunidades.

Todas estas iniciativas se basan en las "creencias" de las poblaciones locales sobre la sacralidad de los respectivos ríos. Aunque los efectos concretos todavía están por definirse, las propias declaraciones significan el reconocimiento de una cierta pluralidad ontológica, si bien puede que las instituciones legislativas o judiciales que han promulgado dichas leyes o dictado las sentencias mencionadas no sean completamente conscientes del alcance de sus declaraciones y todavía se muevan dentro del paradigma del multiculturalismo.

\section{CONCLUSIONES}


Plantear conflictos ontológicos, sea cual sea su naturaleza, es algo que puede parecer contraintuitivo porque obliga a cuestionar un postulado básico de todas las ciencias sociales: el de que las diferencias entre los colectivos humanos son diferentes perspectivas sobre una realidad única (Blaser, 2013b: 547). A pesar de parecer algo muy evidente, dado que está arraigada en el pensamiento occidental moderno, la suposición de que la ciencia agota el conocimiento de la realidad, al mismo tiempo consecuencia y argumento que sustentan el naturalismo, no resiste el menor ejercicio de antropología simétrica. Las descripciones y explicaciones dadas por los diferentes colectivos humanos de sus relaciones con los elementos del mundo no pueden ser reducidas a la división naturaleza-cultura sin perder gran parte de su significado real, es decir, el significado para el grupo que las enuncia.

Como se ha argumentado en el artículo, en el ámbito de la política y el derecho, una mala comprensión de una determinada realidad antropológica puede tener consecuencias negativas directas para los grupos en conflicto. Esta es la principal justificación para el proyecto de una ontología política. Sin herramientas conceptuales y jurídicas capaces de permitir el reconocimiento de las diferentes ontologías, los conflictos de esta naturaleza no pueden gestionarse bien.

Aparte de los efectos positivos para la gestión de los conflictos ontológicos concretos, el reconocimiento político y jurídico del pluralismo ontológico, del pluriverso o del "derecho a la autodeterminación ontológica", como se le quiera denominar, tendría posiblemente un efecto en la crisis ecológica porque, como observa Latour (2012b), el mundo contemporáneo debe elegir entre modernizarse o ecologizarse, es decir, extender el proceso de uniformización y expansión del modo de vida occidental moderno, o crear espacios institucionales para que sea posible resistir a dicho proceso. Reconocer la autonomía conceptual y la necesidad de desarrollar una teoría de los conflictos ambientales ontológicos es caminar hacia la segunda opción.

\section{BIBLIOGRAFÍA}

BLASER, Mario, "The Threat of the Yrmo: The Political Ontology of a 
Sustainable Hunting Program", American Anthropologist, núm. 111 (1), Blackwell Publishing Inc: 10-20, 2009a, doi: 10.1111/j.15481433.2009.01073.x.

— "POLITICAL ONTOLOGY", Cultural Studies, núm. 23 (5-6), Taylor \& Francis Group: 873-96, 2009b, doi: 10.1080/09502380903208023.

— "Notes towards a Political Ontology of 'Environmental' Conflicts”, Contested Ecologies: Dialogues in the South on Nature and Knowledge, Lesley Green (ed.), 13-27, HSRC Press, Cape Town, 2013a.

- "Ontological Conflicts and the Stories of Peoples in Spite of Europe", Current Anthropology, núm. 54 (5). The University of Chicago Press, Wenner-Gren Foundation for Anthropological Research: 547.68, 2013b, doi: 10.1086/672270.

CASTRO, Eduardo Viveiros de, Metafísicas caníbales: líneas de antropología postestructural, Katz Editores, Madrid - Buenos Aries, 2010.

CIRONE, Mariano, "The Vedanta Case in India, EJOLT Factsheet No. 46". Tarragona, 2015. Recuperado de http://www.ejolt.org/wordpress/wpcontent/uploads/2015/08/FS-46.pdf.

CORTE IDH, Caso Comunidad indígena Yakye Axa Vs. Paraguay. Sentencia de 17 de junio de 2005 (Fondo, Reparaciones y Costas). Serie C núm. 125, 2010.

DESCOLA, Philippe, Más allá de naturaleza y cultura, Amorrortu editores, Madrid, 2012.

HARMAN, Graham, Prince of Networks: Bruno Latour and Metaphysics, Prahran, Vic., Re. press, 2009.

HAZARIKA, Utsa, “The Earth Cannot Let Go of Us': Analysing Ontological Conflicts", Ethnos, junio, Routledge, 1-20, 2016, doi: 10.1080/00141844.2016.1171791.

HOLBRAAD, Martin y PEDERSEN, Morten Axel, "Introduction: The Ontological Turn in Anthropology", The Ontological Turn, 1-29, Cambridge University Press, Cambridge, 2017. Recuperado el 15 de junio de 2017, doi: 10.1017/9781316218907.002. 
INDIA, Writ Petition (PIL) No.140 of 2015, 2017.

KUUKKANEN, Jouni-Matti, "Demystification of Early Latour", François, K., Löwe, B., Müller, Th. y Van Kerkhove, B. (eds.), Foundations of the Formal Sciences VII: Bringing Together Philosophy and Sociology of Science, College Publications, Londres, 2011.

LATOUR, Bruno, The pasteurization of France, Harvard University Press, Cambridge, Mass., 1988.

— "WHOSE COSMOS, WHICH COSMOPOLITICS?: Comments on the Peace Terms of Ulrich Beck", Common Knowledge, núm. 10 (3), Duke University Press: 450-62, 2004, doi:10.1215/0961754X-10-3-450.

- Nunca fuimos modernos: ensayo de antropología simétrica, Siglo Veintiuno Editores, Buenos Aires, 2012a.

- Políticas de la naturaleza. Por una democracia de las ciencias, RBA, Barcelona, 2012b.

— An inquiry into modes of existence : an anthropology of the moderns, 2013.

MARTÍNEZ ALIER, Juan, El ecologismo de los pobres: conflictos ambientales y lenguajes de valoración, Icaria, Barcelona, 2011.

MCANALLY, Elizabeth Ann, "Toward a Philosophy of Water: Politics of the Pollution and Damming along the Ganges River", University of North Texas, 2007. Recuperado de http://search.proquest.com/docview/304828010.

NUEVA ZELANDA, Te Awa Tupua (Whanganui River Claims Settlement) Act 2017, 2017. Recuperado de http://www.legislation.govt.nz/act/public/2017/0007/latest/DLM6830851.htm I\#DLM6831459.

POVINELLI, Elizabeth A., "Radical Worlds: The Anthropology of Incommensurability and Inconceivability", Annual Review of Anthropology, núm. 30 (1), Palo Alto, CA 94303-0139, USA, Annual Reviews 4139 El Camino Way, P.O. Box 10139: 319-34, 2001, doi: 10.1146/annurev.anthro.30.1.319. 
REPÚBLICA DE COLOMBIA, Sentencia T-622/16, 2016.

REPÚBLICA DE BENÍN, INTERMINISTERIAL ORDER N0121/MEHU/MDGLAAT/DC/SGM/DGFRN/SA Setting the Conditions for the Sustainable Management of Sacred Forests in the Republic of Benin, Benín, 2012. Recuperado de http://sacrednaturalsites.org/wpcontent/uploads/2014/09/Benin-Sacred-Forest-law-final-English-version2014.pdf.

STENGERS, Isabelle, Cosmopolitics, University of Minnesota Press, Minneapolis, 2010.

- "Accepting the Reality of Gaia: A Fundamental Shift?", Bonneuil, Ch., Gemenne, F. y Hamilton, C., The Anthropocene and the Global Environmental Crisis, 134-44, Routledge, Londres - Nueva York, 2015.

VIVEIROS DE CASTRO, Eduardo, A inconstância da alma selvagem e outros ensaios de antropologia, Cosac \& Naify, São Paulo, 2014.

- "Sobre Os Modos de Existência Dos Coletivos Extramodernos: Bruno Latour E as Cosmopolíticas Ameríndias", Río de Janeiro, 2016. Recuperado de https://www.academia.edu/21559561/Sobre_o_modo_de_existencia_dos_ coletivos_extramodernos.

WAGNER, Roy, The Invention of Culture, University of Chicago Press, Chicago, 1981. 